\title{
Study on the Structure and Control System of Spraying Machine
}

\author{
Shenyan Wang, Cheng Zhou, Baisong Yang, Xin Fang \\ Heilongjiang Academy of Land Reclamation, Haerbin, Heilongjiang, 150038
}

Keywords: Spraying Machine, Control System, Structure Analysis

\begin{abstract}
China's plant protection machinery from scratch, has gone through a long process of development, has made some achievements, but compared with foreign advanced plant protection machinery, still can not meet the current needs of agricultural development, large-scale mechanization level is still not high, the traditional Manual plant protection machinery still occupy the main position. Aiming at the status quo of China's plant protection machinery, this paper designed the plant protection machinery - spraying machine which is mainly used for field spraying.
\end{abstract}

\section{Introduction}

Plant protection is an important component of agricultural production and is one of the necessary measures to ensure crop harvest. In order to control the effects of various diseases, insects and weeds on crops, people have taken the necessary protective measures, in the course of practice, make great efforts to play a positive role in various measures, to minimize the loss of crops. There are many types of plant protection machinery, the style is strange, the traditional small backpack sprayer, advanced large-scale self-propelled spray machine, many places even appeared in the aviation sprayer. According to the different ways of discrimination, plant protection machinery usually have the following categories: according to the different pesticide formulations can be divided into sprayer, spray machine, dusting machine; in accordance with the different supporting power can be divided into manpower, animal machine, small power machine, Large-scale tractor, self-propelled plant protection machine and aircraft equipment as the carrier of the machine; according to the different liquid can be divided into constant spray machine, low-volume sprayer and micro-spray machine; according to the agent can also be divided into atomization Hydraulic machines, pneumatic machines, thermal machines, centrifuges and static motors.

\section{The Overall Structure Design of Spraying Machine}

Spraying machine is a set of machines, electricity, liquid as one of the system, which consists of mechanical structure, hydraulic power system, control system components. If the machine is a human body, the mechanical structure is its skeleton, the hydraulic system is its muscle, the control system is its brain. Therefore, the first step in the design of the sprayer is to design the mechanical structure. Before the design of mechanical structure, has been clear this design of the spray machine has what function: (1) is mainly used for field crops spraying work, operating area to large; (2) according to the different crop spacing to adjust the wheel Position; (3) the height of the nozzle can be adjusted according to the plant height of the crop.

In the design process, the use of a number of sets of hydraulic systems to achieve the cab and spray rack movements, the adjustment of the horizontal spacing of the wheel and the steering of the machine. The engine, fuel tanks, medicine boxes, etc. arranged in the middle of the spraying machine. All kinds of rods, plates are hinged or welded to connect, the hydraulic system in the tubing is not reflected in the figure. When the driver rotates the steering wheel, drives the hydraulic steering gear, connects the steering cylinder, and the hydraulic cylinder drives or pulls the steering arm to rotate about the main pin. The steering arm and the wheel are a whole, thus turning the wheel. The side frame and the top frame are hinged, and the both ends of the hydraulic cylinder are 
arranged on the top frame and the side frame of the spraying machine. Adjust the hydraulic cylinder to extend, to promote the sprayer side frame outward expansion, the wheel horizontal spacing becomes larger, adjust the hydraulic cylinder shrink, pull the side frame inward recovery, the wheel lateral spacing becomes smaller. With this structure, the dispenser can adjust the lateral spacing of the wheels according to the pitch. Using a similar hydraulic system, the use of hydraulic cylinders to achieve the sprayer cab and spray rack movements, adjust the height of the cab and spray rack. When the hydraulic cylinder is extended, the piston rod drives the tripod to rotate inward, and the tripod drives the sprayer arm to rotate inward to realize the shrinkage of the spray frame. When the hydraulic cylinder shrinks, the piston rod pulls the tripod to rotate outward, and the tripod drives the sprayer arm to rotate outward to realize the extension of the spray frame. The use of similar institutions to achieve the expansion of the spray frame arm, not repeat them. This greatly increases the operating radius of the sprayer.

In this case, the shock absorber used in this paper is a hydraulic damper. The shock absorber is always used in conjunction with the spring, when the spring by the impact of vibration, will be repeated compression and rebound, then the shock absorber on the action of the spring damping effect, so that the spring tends to be stable.

The internal part of the hydraulic shock absorber is divided into two parts: the storage tank and the working cylinder. The cylinder is divided into the upper chamber and the lower chamber by the piston. The whole shock absorber is filled with special hydraulic oil. The extension valve and the flow valve are distributed on the piston and are the passage of the oil between the upper and lower chambers of the cylinder. There is a compression valve and a compensation valve between the cylinder and the reservoir, which is the passage between the oil and the oil. At present, the more advanced shock absorber, the key components are the use of resistance welding technology to complete the welding.

Compensation valve and the flow valve is a common one-way valve, spring force is relatively small, only a small oil pressure can be opened, the compression valve and the extension valve is unloading valve, need to reach a certain value after the valve to open. When the shock absorber does not work, under the combined action of oil pressure and spring force, each valve is closed and the hydraulic oil is not circulated. When the shock absorber is compressed, the piston rod to move the piston down, the cylinder chamber volume becomes larger, lower chamber volume becomes smaller, the flow valve opens, the lower chamber fluid into the upper chamber, the piston continues down, the lower chamber pressure Increased to a certain value, the compression valve open, part of the oil into the tank. The throttle effect of the two valves on the oil forms the damping force of the shock absorber when compressed. When the shock absorber is stretched, the piston rod pulls the piston upward movement, the cylinder cavity volume becomes smaller, the lower chamber volume becomes larger, the extension valve opens, the upper chamber fluid enters the lower chamber, the piston continues upward and the lower chamber pressure Down to a certain value, the compensation valve to open, part of the oil from the tank into the lower chamber. The throttle effect of the two valves on the oil forms the damping force of the shock absorber when subjected to stretching. Shock absorber work, the oil flow between the cavity repeatedly, the absorption of mechanical energy into heat, and finally absorbed by the cavity to the atmosphere, and ultimately to achieve damping effect.

Before the work of the spraying machine, according to the different working conditions, to spray the machine to make different adjustments, namely the wheel spacing, the height of the cab and spray frame, spray frame size arm expansion position. Use the hydraulic system to design these four cycles to complete this work. The four loops do not interfere with each other and can act alone. Cycle 1: Adjust the horizontal spacing of the wheels. Widen $\rightarrow$ stop, lock $\rightarrow$ shrink $\rightarrow$ stop. Circle 2: cab lift. Rise $\rightarrow$ stop, lock $\rightarrow$ drop $\rightarrow$ stop. Circulation three: spray rack lift. Rise $\rightarrow$ stop, lock $\rightarrow$ drop $\rightarrow$ stop. Circulation four: spray around the arm at the same time stretching and contraction. Arm at the same time stretching, the arm does not move $\rightarrow$ arm to stop, the arm at the same time stretching $\rightarrow$ arm to stop the action $\rightarrow$ arm contraction, arm does not move $\rightarrow$ arm stop, arm contraction, complete a cycle. When the hydraulic components do not work, the use of 
tightening circuit to cut off the oil into the channel, so that the hydraulic components to maintain a certain stability. In the design process of the spraying machine, the cab and the sprayer are raised to a certain height and then stopped. After the work is started, it is necessary to ensure that the cab and the spray booth are stable during the operation, and can not be beaten due to their own weight or other external factors.

\section{Design of PLC Control System for Spraying Machine}

PLC is the core of the spray control system, the correct choice of PLC is essential. According to the structure category, PLC is generally divided into monolithic and modular, each has its own advantages and disadvantages. Integral PLC is relatively cheap, commonly used in the work environment is better occasions, modular PLC function expansion is relatively easy, easy to fault diagnosis, easy maintenance. PLC I/O modules generally include switching input and output modules, analog input, output modules and other special modules. In the design of the PLC control system of the spraying machine, this article only uses the input and output of the switch quantity, and there is no sensor existence, that is, no analog quantity. According to the control process, PLC control system has 15 input buttons, 10 trip switch, a total of 25 input signals, are the switch volume. The output signal has 12, are used for the control valve solenoid valve.

In summary, select FX2N-64MR PLC, shape shown in Figure 5-2. 2N Mitsubishi PLC is by far the FX family of the most advanced PLC series, with high-speed processing, expansion of a large number of special features such as modules, with a strong flexibility and control. FX2N series is a small, high-speed, high-performance ultra-small program device, is by far the fastest running one of the small PLC. 64 that there are 32 inputs and 32 outputs, fully meet the control requirements, and leave a certain margin. $M$ represents a basic unit.

PLC control system hardware module is completed, I/O address allocation is completed, then the PLC control system software design. PLC program structure is roughly divided into linear structure, modular structure of two forms. The linear structure is to put the user program in a single instruction block, that is, a simple program block contains all the instructions of the system. Such procedures are relatively simple and direct, suitable for the control process is relatively simple, the program is not a particularly complex system. The modular structure classifies processes that require similar or related functions and attempts to provide a common solution that can be used for several tasks. The information is provided to the instruction block, and the modular program can reuse these common modules. Such a design, the program design is very clear, very suitable for control more objects, the control process more complex system. According to the needs of the spraying machine, select the linear structure.

\section{Conclusion}

Most of the overall structure uses rods and plates, through the articulation or welding in the form of the entire assembly machine. The steering mechanism is designed to realize the steering of the spraying machine, the wheel lateral pitch adjustment mechanism is used to adjust the wheel wheel distance to adapt to the different row distance, the lifting system to adjust the height of the cab and the spray frame, The working area of the machine, the hydraulic motor to drive the machine to walk, and the use of shock absorber to improve the smoothness and comfort of the machine. The control system is divided into hydraulic control system and PLC control system. The design used a number of sets of hydraulic cylinders. Hydraulic system in the PLC under the control of the realization of the solenoid valve on and off, and then through the hydraulic cylinder to further promote the movement of various institutions, and ultimately to achieve the requirements of the spraying machine. In the hydraulic system, the design of the lock, sequence, unloading, synchronization, buffer and other circuits, and draw a complete hydraulic system map. PLC is the core of the control system, according to the input and output of the selected FX2N-64MR PLC, combined with the spray machine workflow, drawing out the electrical control circuit, a reasonable allocation of I/O address, drawing the I/O wiring Figure, and the preparation of the PLC control program. 


\section{Acknowledgements}

Key Technology in Heilongjiang Province and Equipment Development of Low Drift Low Dosage Spray Sprayer

Project No : HNK135-03-09

\section{References}

[1] Fu Daping, Tian Yun. China's plant protection machinery development status and prospects analysis [J]. Agriculture and Technology, 2012 (11)

[2] Chen Qiang. Analysis of the development trend of plant protection machinery [J]. Science and Technology Wind, 2010 (24)

[3] Li Lin, Guo Yong. Automotive shock absorber key welding equipment [J]. Welding machine, $2010(05)$

[4] Ministry of Agriculture issued a notice on accelerating the mechanization of plant protection [J]. Pesticide Market Information, 2008 (17)

[5] Wang Jilin, Wang Xueliang.On the agricultural plant protection mechanization [J]. Agricultural Machinery, 2007 (16) 